(c) Institute of Mathematical Statistics, 2018

\title{
Fractionally Differenced Gegenbauer Processes with Long Memory: A Review
}

\author{
G. S. Dissanayake, M. S. Peiris and T. Proietti
}

\begin{abstract}
The main objective of this paper is to review and promote the usefulness of generalized fractionally differenced Gegenbauer processes in time series and econometric research endeavours. In particular, theoretical and computational aspects centered around fractionally differenced Gegenbauer processes with long memory together with a number of interesting and elegant extensions will be discussed. In-depth conceptual developments and large scale simulation study results are presented for clarity and completeness. This survey highlights a number of gaps in the existing literature of this subject area and becomes a valuable reference source for time series practitioners.
\end{abstract}

Key words and phrases: Gegenbauer process, long memory, heteroskedasticity, fractional difference, volatility, spectral density, stationarity, invertibility.

\section{INTRODUCTION}

The origin of stochastic analysis of time series can be traced back to the seminal work of Yule (1926) and Slutsky (1927), who introduced autoregressive (AR) and moving average (MA) models. Box and Jenkins (1970) provided a systematic inferential treatment of stationary ARMA models, which became the workhorse of modern applied time series work. The next decade would set the scene for nonstationarity, integration and fractional integration. In particular, in the early 1980s the introduction of long memory by Granger and Joyeux (1980) and Hosking (1981) opened a new fruitful research avenue for time series specialists and econometricians. The class of fractionally integrated autoregressive moving average (ARFIMA or FARIMA) processes, extending the

G. S. Dissanayake is Senior Lecturer, Statistics, NSBM Green University, Homagama, Sri Lanka and Honorary Associate, School of Mathematics and Statistics, University of Sydney, Sydney NSW, Australia (e-mail: gnanadarshad@gmail.com).M. S. Peiris is Professor of Statistics, School of Mathematics and Statistics, University of Sydney, Sydney NSW, Australia. T. Proietti is Professor, Economics and Statistics, University of Rome Tor Vergata, Rome, Italy and Fellow, Center for Research in Econometric Analysis of Time Series, Aarhus University, Aarhus, Denmark. traditional autoregressive integrated moving average (ARIMA) series with a fractional degree of differencing, is nowadays routinely applied to diverse phenomena such as energy price and demand, telecommunication, environmental time series and financial volatility. A thorough account of the developments and the various approaches to inference are reported in Beran (1992, 1994), Chan and Palma (2006), Giraitis, Koul and Surgailis (2012) and Beran et al. (2013).

The Gegenbauer processes that constitute the topic of this paper were introduced in the last paragraph of Hosking's (1981) seminal Biometrika article. The concept was later developed by Anděl (1986) and Gray, Zhang and Woodward (1989, 1994), who proposed the class of time series models known as Gegenbauer ARMA abbreviated as GARMA, using the theory of Gegenbauer polynomials (see also Giraitis and Leipus, 1995, and Woodward, Cheng and Gray, 1998, for further details). Their distinctive properties are long range dependence and quasi-periodic behaviour, and they provide a model for cyclical or seasonal persistent processes, whose autocorrelation function is an hyperbolically damped oscillating sequence. As a result, the autocorrelation function is not absolutely summable and the spectrum possesses a pole at the cyclical or seasonal frequencies. A detailed analysis of the long memory features of GARMA processes is in Chung 
(1996). As shown by Oppenheim and Viano (2004), cyclical and seasonal persistent processes can arise from the contemporaneous aggregation of stationary autoregressive processes with complex random roots. This generalized class can be used to represent long memory depicting multiple unbounded spectral peaks away from the origin, unlike in the standard long memory ARFIMA case of Hosking (1981), which can only show unbounded spectral density peaks at the zero frequency. A detailed analysis of the long memory features of GARMA processes is in Chung (1996).

In many financial time series modelling problems, it is known that heteroskedasticity plays an important role. Such models in common use are the autoregressive conditionally heteroskedastic (ARCH) model of Engle (1982) and its generalized ARCH (GARCH) model due to Bollerslev (1986). An extension of it by Baillie, Bollerslev and Mikkelsen (1996) resulted in a fractionally integrated GARCH (FI-GARCH) to model the conditional variance. Incorporating heteroskedasticity in ARFIMA models with GARCH errors has been studied by Ling and Li (1997). Another interesting development was the introduction of the Gegenbauer integrated GARCH (GIGARCH) by Guegan (2000) combining the attributes of long memory, seasonality and heteroskedasticity at the same time. In an attempt to capture and blend some of these established GARCH features, Dissanayake and Peiris (2011) introduced conceptual properties of a class of models with conditionally heteroskedastic errors. It was based on a generalized fractional operator used by Anh, Angulo and Ruiz-Medina (1999) and later developed by Peiris (2003), Shitan and Peiris (2008, 2013). This scaled down new operator with further applications was employed by Peiris, Allen and Peiris (2005) and Peiris and Thavaneswaran (2007) in long memory models driven by heteroskedastic GARCH errors.

Furthermore, it has become a customary practice in applied time series analysis to conduct tests to assess whether a time series is stationary or to be integrated at a suitable degree of differencing. This procedure became very popular among econometricians and was made famous under the theme of "unit root hypothesis" (see, e.g., Phillips and Xiao, 1998; Dolado, Gonzalo and Mayoral, 2002). Such hypotheses of stationary series with respect to unit roots could be extended to fractional processes with long memory. A unit root test for fractionally integrated processes has been proposed in Dolado, Gonzalo and Mayoral (2002) and asymptotic results of a similar test is presented in Wang, Lin and Gulati (2003). Additionally, Taylor (2005) introduced a set of new tests to assess constant trend stationarity against the change in persistence from trend stationarity to difference stationarity or vice versa. Furthermore, Ohanissian, Russell and Tsay (2008) proposed a statistical test to distinguish between true and spurious long memory. However, an extension of the unit root test based on state space methodology and Kalman filter (KF) estimates for a GARMA model is seemingly absent in the literature. Jansson and Nielsen (2012) proposed a nearly efficient unit root test that is applicable in such a context and assessing its power with a GARMA model becomes a worthwhile exercise.

In summary, consideration of this paper will be given to a class of Gegenbauer processes generated by Gaussian white noise and GARCH errors since most of the other models are nested within this new family. In other words, this will be a review of two topics relevant to the class known as "fractional differencing" and "long memory". Section 2 provides preliminaries of fractionally differenced Gegenbauer processes with long memory. Gegenbauer processes and the use of truncated state space representations and $\mathrm{KF}$ in estimating and forecasting their long memory version will be considered in Sections 3 and 4. The state space methodology follows the work of Anderson and Moore (1979), Pearlman (1980), Harvey (1989), Aoki (1990), Brockwell and Davis (1996), Chan and Palma (1998), Durbin and Koopman (2001), Harvey and Proietti (2005), Palma (2007), Grassi and Santucci de Magistris (2014) and Dissanayake, Peiris and Proietti (2016). The adoption of the techniques in Dissanayake, Peiris and Proietti $(2016,2015)$ to analyze seasonality in long memory Gegenbauer series is also presented in this section. This will be followed by Section 5 on modelling of persistence and conditional variance. A model testing phase based on Dissanayake et al. (2015) will be presented in Section 6, and Section 7 will comprise of concluding remarks.

\section{PRELIMINARIES}

Let $\left\{X_{t}, t=1,2, \ldots,\right\}$ be a stationary random process with autocovariance function $\gamma(k)=\operatorname{Cov}\left(X_{t}\right.$, $\left.X_{t+|k|}\right)$, and autocorrelation function (acf) $\rho(k)=$ $\gamma(k) / \gamma(0)$, where $k=0,1,2, \ldots$ We denote the normalized spectrum or spectral density function (sdf) by

$$
f(\omega)=\frac{1}{2 \pi} \sum_{k=-\infty}^{\infty} \rho(k) e^{-i \omega k}, \quad-\pi \leq \omega \leq \pi,
$$

where $\omega$ is the Fourier frequency. 
According to the behaviour of the spectral density and/or the acf, we can characterise the notion of long range dependence. The following details of a long memory process will be adopted in the sequel. As it is illustrated by Guégan (2005) and Giraitis, Koul and Surgailis (2012), Chapter 3, there are several alternative characterisations of the notion of long memory. In this paper, we will adopt a definition that is based on the second-order properties, and in particular on the asymptotic properties of the autocorrelation according to which $\left\{X_{t}, t=1, \ldots, T\right\}$ is said to be long memory if $\sum_{k=0}^{T-1}|\rho(k)| \rightarrow \infty$ as $T \rightarrow \infty$. The following definition specializes the role of decay of $\rho(k)$ at infinity.

Long Memory Cyclical Process: $\left\{X_{t}\right\}$ is a long memory process at the frequency $\omega_{0} \in[0, \pi]$ if $\rho(k) \sim$ $C_{\rho} k^{2 \delta-1} \cos \left(k \omega_{0}\right)$ as $k \rightarrow \infty$, where $\delta \in(0,0.5)$ is called the memory parameter and $C_{\rho}>0$.

Here, given two sequences $a_{k}$ and $b_{k}, a_{k} \sim b_{k}$ means $\lim _{k \rightarrow \infty} \frac{a_{k}}{b_{k}}=1$. By Lemma 7.3.1 in Giraitis, Koul and Surgailis (2012), if the spectral density of $\left\{X_{t}\right\}$ takes the form $f(\omega) \sim C(\omega)\left|\omega-\omega_{0}\right|^{-2 \delta}$, where $C(\omega)$ is a regularly varying function with $\lim _{\omega \rightarrow \omega_{0}} h(\omega)=$ $h\left(\omega_{0}\right)>0$, and derivative satisfying $\int_{-\pi}^{\pi} \frac{d C(\omega)}{d c} d \omega<$ $\infty$ and $\left(\omega-\omega_{0}\right) \frac{d C(\omega)}{d c} d \omega=0$, for $\omega \rightarrow \omega_{0}$. Then $\left\{X_{t}\right\}$ is long memory according to the above definition with $C_{\rho}=2 C\left(\omega_{0}\right) \Gamma(1-2 \delta) \sin (\pi \delta)$.

We focus on the class of linear causal processes, according to which $X_{t}$ can be represented as $X_{t}=$ $\sum_{j=0}^{\infty} \psi_{j} \epsilon_{t-j}$, where $\epsilon_{t} \sim \mathrm{WN}\left(0, \sigma^{2}\right)$ (WN stands for white noise), and the coefficients $\psi_{j}$ decay hyperbolically, $\psi_{j} \sim C_{\psi} j^{\delta-1}, j \rightarrow \infty, C_{\psi} \neq 0$. This class could be obtained by introducing the fractional differencing operator and nests important models, such as the autoregressive fractionally integrated moving average (ARFIMA) model, the Gegenbauer ARMA (GARMA) model and the fractional exponential (FEXP) model. The ARFIMA class was introduced by Granger and Joyeux (1980) and Hosking (1981), while Robinson (1991) and Beran (1993) introduced the FEXP model.

The most elementary fractional linear process featuring long memory at the zero frequency is $(I-B)^{\delta} X_{t}=$ $\epsilon_{t}, 0<\delta<0.5$, which is generalised by replacing $\epsilon_{t}$ by a short memory $\operatorname{ARMA}(p, q)$ process, or by a process with a logarithmic spectrum that can be represented by a finite trigonometric polynomial (FEXP model). The operator $(I-B)^{\delta}$ is the fractional differencing operator and for $\delta>-1$ it is defined according to the binomial expansion

$$
(I-B)^{\delta}=\sum_{j=0}^{\infty} \frac{\Gamma(j-d)}{\Gamma(j+1) \Gamma(-d)} B^{j},
$$

where $\Gamma(z)=\int_{0}^{\infty} x^{z-1} e^{-x} d x$ is the Gamma function.

\section{GEGENBAUER PROCESSES AND THEIR PROPERTIES}

A Gegenbauer process is a long memory process generated by the dynamic equation

$$
\left(1-2 u B+B^{2}\right)^{\delta} X_{t}=\epsilon_{t},
$$

where $|u|<1, \delta \in(0,0.5)$, and $\epsilon_{t}$ is a short memory process characterised by a positive and bounded spectral density $f_{\epsilon}(\omega)$. If $\epsilon_{t} \sim \mathrm{WN}\left(0, \sigma^{2}\right)$, (1) is a well-known Gegenbauer process of order $\delta$ or a $\operatorname{GARMA}(0, \delta, 0)$ process. Some authors use the notation $\operatorname{GARMA}(0, \delta, 0 ; u)$ to represent the process in (1). See, for example, Peiris and Asai (2016). As we shall see below, (1) complies with the definition given in Section 2 of a long memory process at the frequency $\omega_{0}=\arccos (u)$. According to (1), $X_{t}$ arises from filtering the process $\epsilon_{t}$ by the infinite impulse response filter

$$
\left(1-2 u B+B^{2}\right)^{-\delta}=\sum_{j=0}^{\infty} C_{j}^{\delta}(u) B^{j},
$$

which we now characterise.

The Gegenbauer or ultraspherical polynomial $C_{j}^{\delta}(u)$ is defined in terms of its generating function as follows:

$$
\frac{1}{\left(1-2 u z+z^{2}\right)^{\delta}}=\sum_{j=0}^{\infty} C_{j}^{\delta}(u) z^{j},
$$

where

$$
\begin{aligned}
C_{j}^{\delta}(u)= & \sum_{k=0}^{[j / 2]}(-1)^{k} \\
& \cdot \frac{\Gamma(j-k+\delta)}{\Gamma(\delta) \Gamma(j+1) \Gamma(j-2 k+1)}(2 u)^{j-2 k}
\end{aligned}
$$

and $[j / 2]$ stands for the integer part of $\frac{j}{2}$ (see Erdelyi et al., 1953, 10.9).

It is possible to show (see Gould, 1974, Gradshteyn and Ryzhik, 1980 and Rainville, 1960, for details) that $C_{j}^{\delta}(u)$ satisfies the recursion

$$
\begin{aligned}
C_{j}^{\delta}(u)= & \frac{1}{j}\left[2 u(j+\delta-1) C_{j-1}^{\delta}(u)\right. \\
& \left.-(j+2 \delta-2) C_{j-2}^{\delta}(u)\right],
\end{aligned}
$$

for $j \geq 2$, with $C_{0}^{\delta}(u)=1$ and $C_{1}^{\delta}(u)=2 \delta u$.

For large $j$, the coefficients $C_{j}^{\delta}(u)$ can be approximated by (see Chung, 1996)

$$
C_{j}^{\delta} \sim\left(\frac{j}{2}\right)^{\delta-1} \frac{\cos \left[(j+\delta) \omega_{0}-(\delta \pi / 2)\right]}{\Gamma(\delta) \sin ^{\delta}\left(\omega_{0}\right)} .
$$


As we have assumed $|u|<1$, the polynomial $1-$ $2 u z+z^{2}$ has complex zeros which are on the unit circle. Therefore, we need the additional restriction $\delta<1 / 2$ to ensure the square summability of $C_{j}^{\delta}(u)$, or $\sum_{j=0}^{\infty}\left\{C_{j}^{\delta}(u)\right\}^{2}<\infty$.

If $\epsilon_{t} \sim \mathrm{WN}\left(0, \sigma^{2}\right)$, the spectral density of $X_{t}$ is

$$
\begin{aligned}
f(\omega) & =\frac{\sigma^{2}}{\pi}\left\{2\left|\cos (\omega)-\cos \left(\omega_{0}\right)\right|\right\}^{-2 \delta} \\
& =\frac{\sigma^{2}}{\pi}\left|4 \sin \left(\frac{\omega+\omega_{0}}{2}\right) \sin \left(\frac{\omega-\omega_{0}}{2}\right)\right|^{-2 \delta}
\end{aligned}
$$

so that, as $\omega \rightarrow \omega_{0}$,

$$
f(\omega) \sim \frac{\sigma^{2}}{\pi}\left\{2\left(\omega-\omega_{0}\right) \sin \left(\omega_{0}\right)\right\}^{-2 \delta} .
$$

Since $\sin \left(\omega_{0}\right)$ is bounded, it is clear that the spectral density is unbounded as $\omega \rightarrow \omega_{0}$. Therefore, it can be shown that a stationary Gegenbauer process contains and unbounded spectrum at $\omega_{0}$ and is long memory when $0<\delta<\frac{1}{2}$. This special frequency $\omega_{0}$ is called the Gegenbauer or $G$-frequency.

Given the spectral density $f(\omega)$, one can compute the autocovariance function $\gamma(j)$ for a stationary long memory Gegenbauer process. For the case $|u|<1$, Gray, Zhang and Woodward (1989) have shown that

$$
\begin{aligned}
\gamma(j)= & \frac{\sigma^{2}}{2 \sqrt{(\pi)}} \Gamma(1-2 \delta)\left[2 \sin \left(\omega_{0}\right)\right]^{1 / 2-2 \delta} \\
& \cdot\left[P_{j-1 / 2}^{2 \delta-1 / 2}(u)+(-1)^{j} P_{j-1 / 2}^{2 \delta-1 / 2}(-u)\right],
\end{aligned}
$$

where $P_{a}^{b}(x)$ are associated Legendre functions that can be calculated using the recursion

$$
P_{a}^{b}(x)=\frac{2 a-1}{a-b} x P_{a-1}^{b}(x)-\frac{a+b-1}{a-b} P_{a-2}^{b}(x),
$$

with initial terms

$$
\begin{aligned}
P_{-1 / 2}^{2 \delta-1 / 2}(u)= & {[(1+u) /(1-u)]^{\delta-1 / 4}[1 / \Gamma(3 / 2-2 \delta]} \\
& \cdot F(1 / 2,1 / 2 ; 3 / 2-2 \delta ;(1-u) / 2), \\
P_{1 / 2}^{2 \delta-1 / 2}(u)= & {[(1+u) /(1-u)]^{\delta-1 / 4}[1 / \Gamma(3 / 2-2 \delta]} \\
& \cdot F(-1 / 2,1 / 2 ; 3 / 2-2 \delta ;(1-u) / 2),
\end{aligned}
$$

and

$$
F(a, b ; c ; w)=\sum_{n=1}^{\infty} \frac{\Gamma(c) \Gamma(a+n) \Gamma(b+n)}{\Gamma(a) \Gamma(b) \Gamma(c+n) \Gamma(n+1)} w^{n}
$$

is the hypergeometric function. This leads towards the following approximation on autocovariances:

$$
\begin{aligned}
\gamma(j)= & \frac{2^{1-2 \delta} \sigma^{2}}{\pi} \sin ^{-2 \delta}\left(\omega_{0}\right) \sin (\delta \pi) \\
& \cdot \Gamma(1-2 \delta) \cos \left(j \omega_{0}\right) \frac{\Gamma(j+2 \delta)}{\Gamma(j+1)} .
\end{aligned}
$$

The acf of $\left\{X_{t}\right\}$ can be approximated by $\rho(j) \sim$ $K \cos \left(j \omega_{0}\right) j^{2 \delta-1}$ as $j \rightarrow \infty$, where $K$ is a real constant that depends on $\delta$ and $\omega_{0}$; see Chung (1996).

When $|u|=1$, that is, $\omega_{0}=0, \pi$, then

$$
C_{j}^{\delta}(u)=(-1)^{j} \frac{\Gamma(j+2 \delta)}{\Gamma(2 \delta) \Gamma(j+1)} ;
$$

the roots of the polynomial $1-2 u z+z^{2}$ are \pm 1 with multiplicity 2 and $\delta$ must satisfy $\delta<\frac{1}{4}$ to ensure the square summability of the coefficients. In this case, $\rho(j) \sim K j^{4 \delta-1}$ as $j \rightarrow \infty$.

\section{1 $\operatorname{GARMA}(p, \delta, q)$ Process}

A process $\left\{X_{t}\right\}$ generated by the following equation is called a $\operatorname{GARMA}(p, \delta, q)$ or $\operatorname{GARMA}(p, \delta, q ; u)$ :

$$
\phi(B)\left(1-2 u B+B^{2}\right)^{\delta} X_{t}=\theta(B) \epsilon_{t},
$$

where $\phi(B)$, and $\theta(B)$ are stationary AR and invertible MA operators, respectively, with no common zeros, $\delta$ is the memory parameter and $|u| \leq 1$ is a constant to satisfy (a) $|u|<1$ for $\delta<1 / 2$ or (b) $|u|=1$ for $\delta<$ $1 / 4$.

\subsection{The $k$-Factor Gegenbauer Processes}

Extending the process in 1 , a $k$-factor Gegenbauer process is given by

$$
\prod_{j=1}^{k}\left(1-2 u_{j} B+B^{2}\right)^{\delta_{j}} X_{t}=\epsilon_{t} .
$$

Woodward, Cheng and Gray (1998) illustrate many important properties of this $k$-factor Gegenbauer process. In particular, the spectrum of (18) is given by

$$
\begin{aligned}
f(\omega)= & \frac{\sigma^{2}}{2 \pi} \prod_{j=1}^{k} \mid 1-2 u_{j} \exp (-i \omega) \\
& +\left.\exp (-2 i \omega)\right|^{-2 \delta_{j}},
\end{aligned}
$$

where $0<\omega<\pi$.

By trigonometric identities, we can write

$$
f(\omega)=\sigma^{2} / 2 \pi \prod_{j=1}^{k}\left\{4\left[\cos (\omega)-\cos \left(\omega_{0 j}\right)\right]^{2}\right\}^{-\delta_{j}}
$$


where $\cos \left(\omega_{0 j}\right)=u_{j}, j=1,2, \ldots, k$. Hence, the spectrum has $k$ unbounded peaks at corresponding Gegenbauer frequencies $\omega_{0 j}, j=1, \ldots, k$. In summary, the $k$-factor Gegenbauer process is:

- stationary if (a) $\left|u_{i}\right|<1$ and $\delta_{i}<1 / 2$ or (b) $\left|u_{i}\right|=1$ and $\delta_{i}<1 / 4$, for each $i=1, \ldots, k$;

- stationary with long memory if (a) $\left|u_{i}\right|<1$ and $0<$ $\delta_{i}<1 / 2$ or (b) $\left|u_{i}\right|=1$ and $0<\delta_{i}<1 / 4$, for each $i=1, \ldots, k$.

Woodward, Cheng and Gray (1998) pointed out that the true autocorrelation structure of a $k$-factor Gegenbauer process could be obtained through the inverse Fourier transform of its spectrum $f(\omega)$. Forecasting with the $k$-factor Gegenbauer processes is dealt with in Ferrara and Guegan (2001), whereas Guégan (2005) provides further insight on the properties of the $k$ factor $\operatorname{GARMA}(0, \delta, 0)$ process. The estimation of time-varying long memory parameters of a locally stationary k-factor Gegenbauer process utilizing the wavelet method is presented in Lu and Guegan (2011).

3.2.1 Other generalized fractional processes. The first generalization of traditional ARMA models has dealt with fractional powers of the AR and MA operators. Two processes in particular have emerged as firstand second-order generalized fractional operators and their applications to time series.

- Consider the process $\left\{X_{t}\right\}$ generated by

$$
\begin{aligned}
& (I-\alpha B)^{\delta} X_{t}=\epsilon_{t} ; \\
& \quad-1<\alpha<1 ; \delta>0 ;\left\{\epsilon_{t}\right\} \sim \mathrm{WN}\left(0, \sigma^{2}\right) .
\end{aligned}
$$

This process has been introduced by Spolia, Chandler and O'Connor (1980) under the name 'fractional order equal root autoregressive model', as mentioned by Hosking (1981). The theory was further developed by Gonçalves (1987), Anh, Lunney and Peiris (1997) and Shitan and Peiris (2008, 2013). Clearly, (10) encompasses the standard first-order autoregressive model when $\delta=1$, as well as the fractional noise process when $\alpha=1$ and $0<\delta<$ $1 / 2$.

- Similarly, the second-order fractional autoregressive model arises from generalizing the $\mathrm{AR}(2)$ and is given by

$$
\begin{gathered}
\left(1-\alpha_{1} B-\alpha_{2} B^{2}\right)^{\delta} X_{t}=\epsilon_{t}, \\
\left\{\epsilon_{t}\right\} \sim \mathrm{WN}\left(0, \sigma^{2}\right),
\end{gathered}
$$

where $1-\alpha_{1} z-\alpha_{2} z^{2} \neq 0$ for all $|z| \leq 1, \delta>0$ (see Shitan and Peiris, 2009, for details).
In (11), it is known that, for $\delta=1$ conditions $\alpha_{2}+\alpha_{1}<1, \alpha_{2}-\alpha_{1}<1$ and $-1<\alpha_{2}<1$ must be satisfied by $\alpha_{1}$ and $\alpha_{2}$ to ensure stationary solutions.

Let $1-\alpha_{1} B-\alpha_{2} B^{2}=\left(1-\xi_{1} B\right)\left(1-\xi_{2} B\right)$, where $\xi_{1}+\xi_{2}=\alpha_{1}$ and $\xi_{1} \xi_{2}=-\alpha_{2}$.

By writing (11) as

$$
\left[\left(1-\xi_{1} B\right)\left(1-\xi_{2} B\right)\right]^{\delta} X_{t}=\epsilon_{t},
$$

and following Shitan and Peiris (2009), it can be shown that the solution to (11) can be expressed as

$$
X_{t}=\sum_{k=0}^{\infty} \sum_{l=0}^{\infty} \frac{\Gamma(k+\delta) \Gamma(l+\delta) \xi_{1}^{k} \xi_{2}^{l}}{\Gamma(k+1) \Gamma(l+1) \Gamma^{2}(\delta)} \epsilon_{t-k-l},
$$

where $\Gamma(\cdot)$ is the gamma function satisfying $\Gamma(k)=$ $(k-1)$ ! for integers $k \geq 1$.

The model in (11) is called the generalized second-order autoregression with index $\delta$. For simplicity, we call it Generalized Autoregressive of order 2 [GAR(2)] model. It is clear that the class generated by (11) is more flexible than the standard AR(2) model. Although the autocorrelation function of the GAR(2) model can be obtained, it is not mathematically tractable as there is no closed form solution. The spectral density $f(\omega)$ is given by

$$
\begin{aligned}
f(\omega)= & \frac{\sigma^{2}}{2 \pi}\left[\left(1+\alpha_{1}^{2}+\alpha_{2}^{2}\right)\right. \\
& \left.-2 \alpha_{1}\left(1-\alpha_{2}\right) \cos \omega-2 \alpha_{2} \cos 2 \omega\right]^{-\delta} .
\end{aligned}
$$

See Shitan and Peiris (2009) for details.

It is clear that when $\alpha_{2}=-1$ [in (11)], the roots of $1-\alpha_{1} z-\alpha_{2} z^{2}=0$ do not lie in the stationary region. In particular, when $\alpha_{1}=2 u,|u|<1$ and $\alpha_{2}=-1$, the zeros of $1-\alpha_{1} z-\alpha_{2} z^{2}$ are a pair of complex conjugate roots having unit modulus with phase $\pm \arccos (u)$ and the process becomes stationary only for particular values of $\delta$. This leads to Gegenbauer processes and extends to a mathematically elegant class of time series models with very useful applications. Now we look at this new class of models.

\section{STATE SPACE REPRESENTATION OF GARMA MODELS}

Consider the Wold representation of a Gaussian $\operatorname{GARMA}(0, \delta, 0)$ process in (1) with $\epsilon_{t} \sim \operatorname{IIDN} \mathrm{N}\left(0, \sigma^{2}\right)$ given by

$$
X_{t}=C(B) \epsilon_{t}=\sum_{j=0}^{\infty} C_{j} \epsilon_{t-j}, \quad C_{0}=1,
$$


where $C(B)=\left(1-2 u B+B^{2}\right)^{-\delta}$ and $C_{j}$ are the Gegenbauer coefficients as defined by (2), where we drop reference to $\delta$ and $u$ for notational simplicity.

The $m$ th order moving average approximation is obtained by truncating the right-hand side of (12) at lag $m$ such that

$$
X_{t, m}=\sum_{j=0}^{m} C_{j} \epsilon_{t-j}, \quad \epsilon_{t} \sim \text { i.i.d. } \mathrm{N}\left(0, \sigma_{m}^{2}\right),
$$

where $\left\{X_{t, m}\right\}$ is the truncated Gegenbauer process that will vary with the chosen finite truncation lag order $m=1,2, \ldots, M<\infty$.

Following Chan and Palma (1998), Dissanayake, Peiris and Proietti (2016) focus on the following state space representation of the above $m$ th order MA approximation:

$$
\begin{aligned}
& X_{t, m}=Z \alpha_{t}+\epsilon_{t}, \\
& \alpha_{t+1}=T_{0} \alpha_{t}+H \epsilon_{t},
\end{aligned}
$$

where $\alpha_{t+1}=[X(t+1 \mid t), X(t+2 \mid t), X(t+3 \mid t), \ldots$, $X(t+m \mid t)]^{\prime}$ is the $m \times 1$ state vector with elements $X(t+j \mid t)=E\left(X_{t+j, m} \mid \mathcal{F}_{t}\right), j=1,2, \ldots, m, \mathcal{F}_{t}=$ $\left\{X_{t, m}, X_{t-1, m}, \ldots,\right\}$, and

$$
\begin{aligned}
Z & =[1,0, \ldots, 0], \\
T_{0} & =\left[\begin{array}{ccccc}
0 & 1 & 0 & \cdots & 0 \\
0 & 0 & 1 & \ddots & 0 \\
\vdots & \vdots & \ddots & \ddots & 0 \\
\vdots & \ldots & \cdots & 0 & 1 \\
0 & 0 & \cdots & \cdots & 0
\end{array}\right], \quad H=\left[\begin{array}{c}
C_{1} \\
C_{2} \\
\vdots \\
\vdots \\
C_{m}
\end{array}\right] .
\end{aligned}
$$

The measurement equation is essentially $X_{t, m}=$ $\mathrm{E}\left(X_{t, m} \mid \mathcal{F}_{t-1}\right)+\epsilon_{t}$, whereas the transition equation provides the predictors $X(t+j \mid t)=X(t+j-1 \mid t)+$ $C_{j} \epsilon_{t}, j=1, \ldots, m$. The distribution of the initial state vector is $\alpha_{1} \sim \mathrm{N}\left(0, P_{1}\right)$, where $P_{1}$ is the Toeplitz matrix with elements $p_{h k}=\sigma_{m}^{2} \sum_{j} C_{j} C_{j+|h-k|}$.

Alternatively, the MA approximation could be obtained by applying the innovations algorithm, following Brockwell and Davis (1991). A different asymptotically equivalent approximation can be obtained using the corresponding truncated $\operatorname{AR}\left(m^{\prime}\right)$ approximation $\sum_{j=0}^{m^{\prime}} \pi_{j} X_{m, t-j}=\epsilon_{t}$, arising from truncating the infinite AR polynomial $\pi(B)=\left(1-2 u B+B^{2}\right)^{\delta}$ at lag $m^{\prime}$. Details can be found in Chan and Palma (1998) and Grassi and Santucci de Magistris (2014), together with a comparison of these two approximations for fractionally integrated processes at the zero frequency. An alternative AR approximation can also be obtained by applying the Durbin-Levinson algorithm to the autocovariance matrix of the process.

\subsection{Estimation and Prediction}

The Kalman filter, Kalman (1961) and Kalman and Bucy (1961), produces recursively the one-step-ahead predictor of $X_{t m}$, given a particular value of the parameter vector, and computes the innovations $v_{t}=$ $X_{m t}-X(t \mid t-1)$, the prediction error variance and the one-step-ahead predictor of the states, as well as their mean square error matrix. Therefore, it enables the evaluation of the likelihood function of the approximating model. The latter can be maximised with respect to the unknown parameters $u$ and $\delta$ (the parameter $\sigma_{m}^{2}$ can be concentrated out of the likelihood function). Let $\left\{x_{t}, t=1, \ldots, T\right\}$ be a time series generated by a Gegenbauer process. The maximiser can be characterised as a quasi maximum likelihood estimator (QMLE) as it converges to the MLE, under regularity assumptions that are detailed below and that entail letting $m$ increase as $T$ increases, at a suitable rate, so that $m / T \rightarrow 0$.

The KF recursions for the approximating $\mathrm{MA}(m)$ model considered in the previous subsection are, for $t=1, \ldots, T$ :

$$
\begin{aligned}
v_{t} & =x_{t}-Z a_{t}, \\
f_{t} & =Z P_{t} Z^{\prime}+\sigma_{m}^{2}, \\
K_{t} & =\left(T_{0} P_{t} Z^{\prime}+\sigma_{m}^{2} H\right) / f_{t}, \\
a_{t+1} & =T_{0} a_{t}+K_{t} v_{t}, \\
P_{t+1} & =T_{0} P_{t} T_{0}^{\prime}+H H^{\prime}-f_{t} K_{t} K_{t}^{\prime},
\end{aligned}
$$

where $K_{t}$ is the Kalman gain, and $f_{t}$ the one-stepahead prediction error variance. The KF returns the pseudo-innovations $v_{t}$, such that if the $\mathrm{MA}(m)$ approximation were the true model, $v_{t} \sim \operatorname{IIDN}\left(0, \sigma_{m}^{2} f_{t}\right)$, so that the quasi log-likelihood of $\left(\delta, u, \sigma_{m}^{2}\right)$ is (apart from a constant term)

$$
\begin{aligned}
& \ell\left(\delta, u, \sigma_{m}^{2}\right) \\
& \quad=-\frac{1}{2}\left(T \ln \sigma_{m}^{2}+\sum_{t=1}^{T} \ln f_{t}+\frac{1}{\sigma_{m}^{2}} \sum_{t=1}^{T} \frac{v_{t}^{2}}{f_{t}}\right) .
\end{aligned}
$$

The scale parameter $\sigma_{m}^{2}$ can be concentrated out of the likelihood function, giving the MLE

$$
\hat{\sigma}_{m}^{2}=\sum_{t} \frac{v_{t}^{2}}{f_{t}} .
$$


The profile log-likelihood

$$
\ell_{\sigma_{m}^{2}}(\delta, u)=-\frac{1}{2}\left[T\left(\ln \hat{\sigma}_{m}^{2}+1\right)+\sum_{t=1}^{T} \ln f_{t}\right]
$$

is a function of parameters $(\delta, u)$.

The maximisation of (17) can be performed by a quasi-Newton algorithm, after a reparameterization which constrains $\delta$ and $u$ in the subset of $\mathbb{R}^{2}[0,0.5) \times$ $[0,1)$.

\subsection{An Illustrative Example}

We illustrate the above state space approach by fitting a Gaussian Gegenbauer process by QMLE to a time series of length $T=512$ generated according to the following mechanism. We simulate $T+m_{0}$ draws from an i.i.d. sequence $\epsilon_{t} \sim N(0,1), t=-m_{0}+$ $1,-1,0,1, \ldots, T$, and we construct the series $\left\{X_{t}\right\}$; $t=1,2, \ldots, T$, using (13), with $u=0.8, \delta=0.4$, $\sigma^{2}=1$. The generated series (for a reasonably large $m_{0}$, such as $m_{0}=10^{3}$ ) can be thought as a realisation of the stochastic process in (12).

The simulated series is plotted in Figure 1 along with its sample autocorrelation function. The latter is a damped sinusoidal with a period of about 10 observations. Figure 2 shows the true spectral density of the Gegenebauer process, $f(\omega)$, which is unbounded at $\omega=\cos ^{-1}(u)=\cos ^{-1}(0.8)$, along with the periodogram of the simulated series, $I\left(\omega_{j}\right)=\frac{1}{2 \pi}[\hat{\gamma}(0)+$ $\left.2 \sum_{k=1}^{T-1} \hat{\gamma}(k) \cos \left(\omega_{j} t\right)\right]$, where $\hat{\gamma}(k)$ is the sample autocovariance function at lag $k$ and $\omega_{j}=2 \pi j / T, j=$ $0, \ldots,\lfloor T / 2\rfloor$, are the Fourier frequencies.
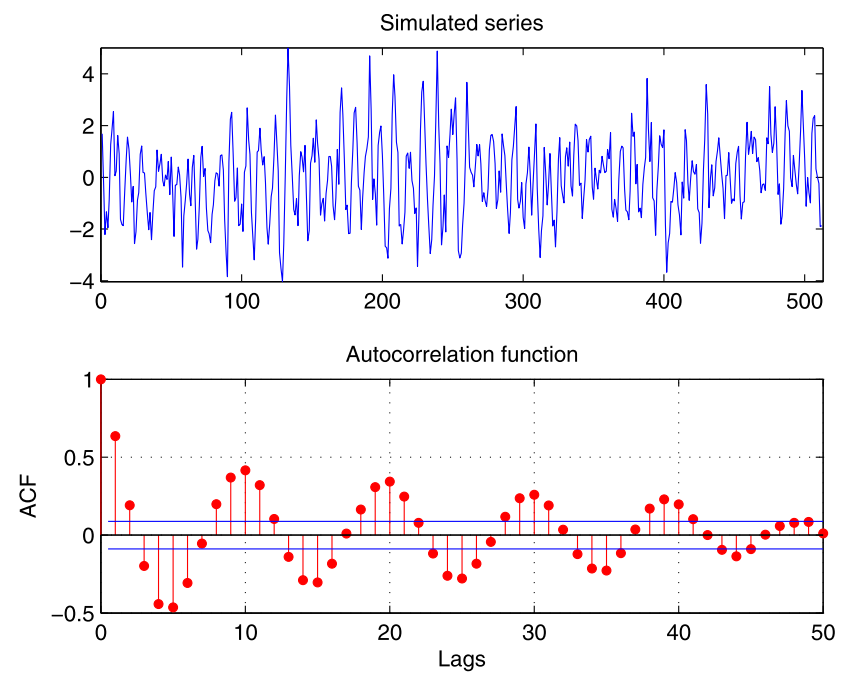

FIG. 1. Simulated series from a Gaussian Gegenbauer process with $\delta=0.4, u=0.8, \sigma^{2}=1$ and corresponding acf.

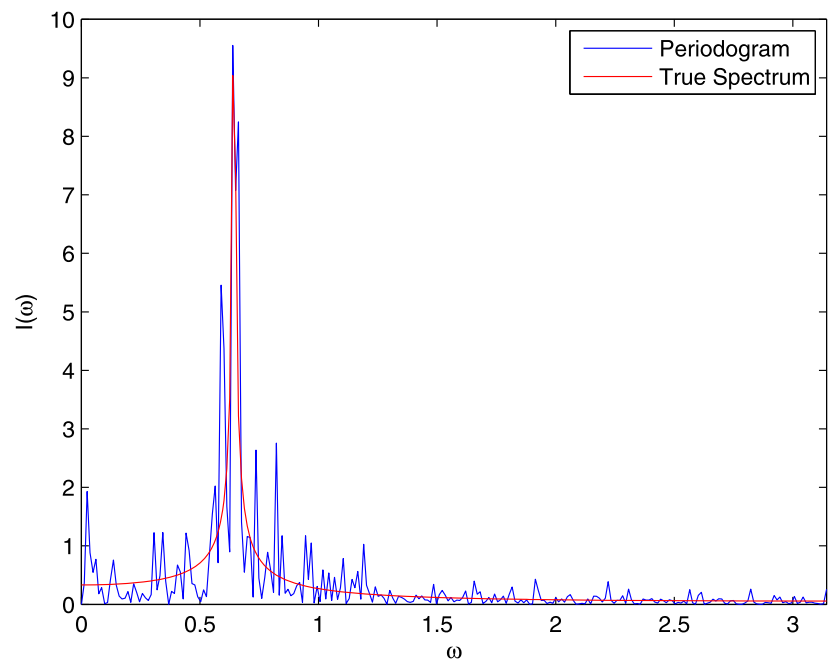

FIG. 2. Sample spectrum (periodogram) and true spectrum of a simulated GARMA $(0, \delta, 0)$ model.

Various $m$ th order truncated MA approximating models have been fitted to the series by the approximate maximum likelihood method outlined in the previous section. The QMLEs of $\delta, u$ and $\sigma^{2}$ are reported in Table 1 . The likelihood is a monotonically increasing function of $m$, whereas the estimated prediction error variance decreases with $m$. On the other hand, $\hat{\delta}$ and $\hat{u}$ vary sensibly with $m$.

Figure 3 displays the implied spectral density of $X_{t, m}$ corresponding to the above parameter estimates. For $m>1$, they are characterised by a spectral peak around the frequency $\cos ^{-1}(\hat{u})$. Another distinguishing feature is the presence of side lobes, due to the truncation of the MA filter (this is referred to as the Gibbs phenomenon).

If the autoregressive approximation is used in lieu of the MA one, the estimated spectral density do not suffer from the Gibbs phenomenon. This is illustrated in Figure 4, which displays the AR spectrum estimates for the same values of $m$.

TABLE 1

Fitting a $\operatorname{GARMA}(0, \delta, 0)$ model to a simulated series using different $\mathrm{MA}(m)$ approximating state space models. Quasi maximum likelihood estimates of the parameters (true values are $\left.\delta=0.4, u=0.8, \sigma^{2}=1\right)$

\begin{tabular}{lccccc}
\hline & \multicolumn{5}{c}{ Values of $\boldsymbol{m}$} \\
\cline { 2 - 6 } & $\mathbf{1}$ & $\mathbf{5}$ & $\mathbf{1 5}$ & $\mathbf{2 5}$ & $\mathbf{5 0}$ \\
\hline$\hat{\delta}$ & 0.32 & 0.41 & 0.50 & 0.44 & 0.43 \\
$\hat{u}$ & 0.98 & 0.80 & 0.75 & 0.78 & 0.78 \\
$\hat{\sigma}_{m}^{2}$ & 1.31 & 1.06 & 0.97 & 0.95 & 0.96 \\
\hline
\end{tabular}




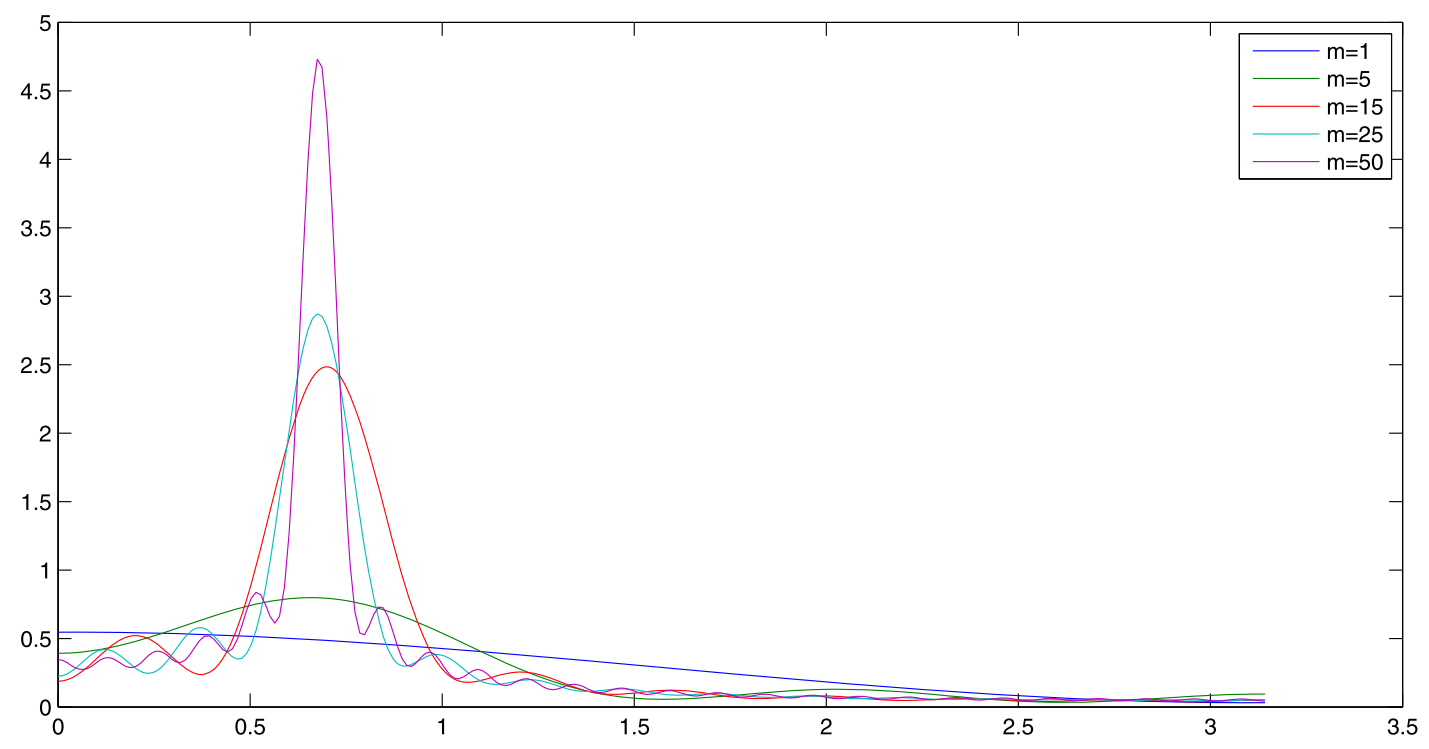

FIG. 3. Spectral densities of estimated MA approximating GARMA $(0, \delta, 0)$ models with different lag orders.

Dissanayake, Peiris and Proietti (2016) present the results of an extensive Monte Carlo experiment, which enabled them to conclude that the optimal order of the MA approximation falls in the range [29, 35], whereas for the AR approximation the optimal truncation is in the range $[9,13]$. The optimality is assessed with respect to the total parameter estimation mean squared error and by the mean square forecast error. Their study also confirmed that the variance of the QMLE of the long memory parameter $\delta$ is $\frac{\pi^{2}}{24 T}$. A further development illustrated that the QMLE approach based on the truncated MA or AR representation outperformed traditional ARIMA and AR models for time series, such

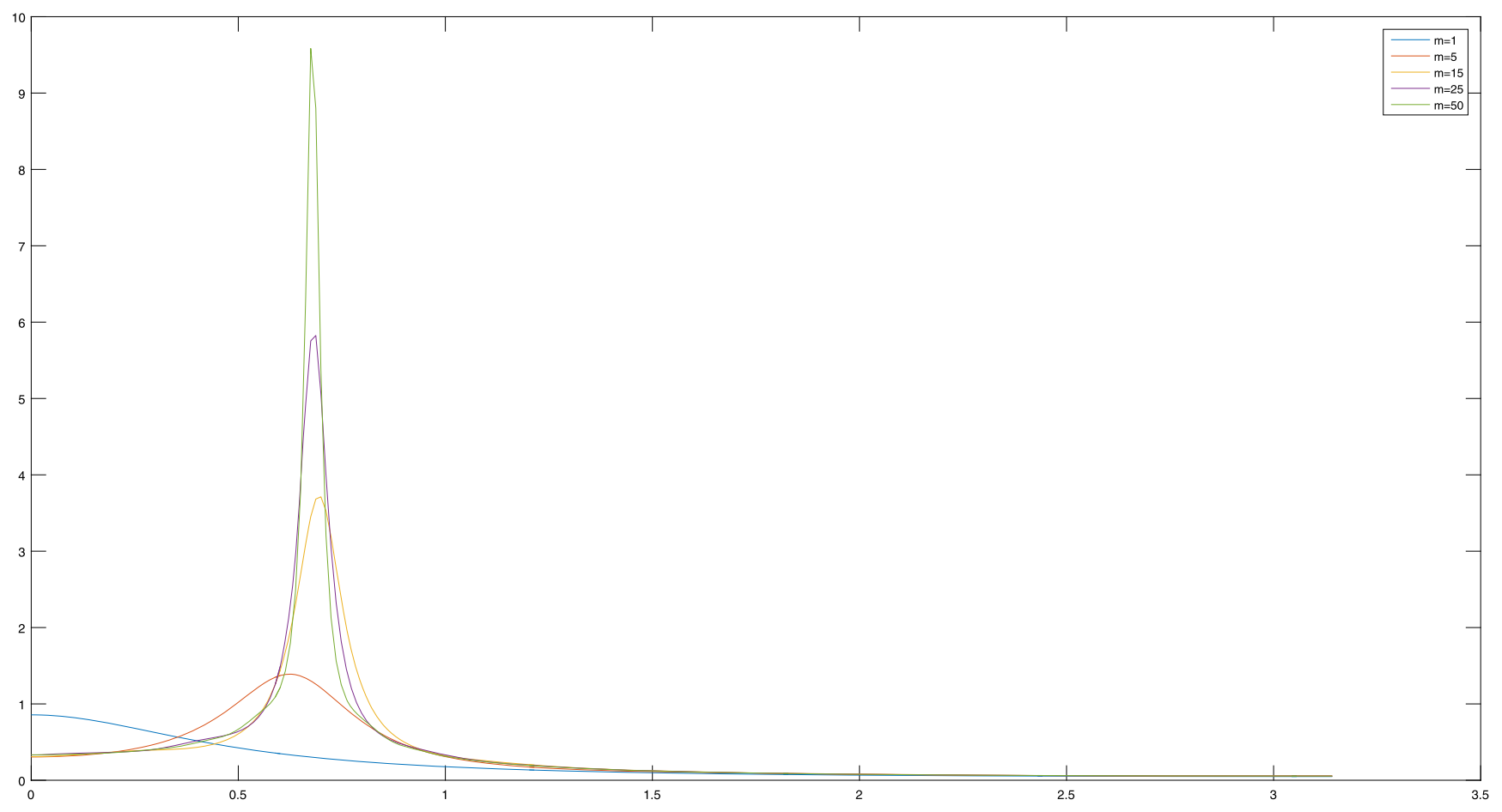

FIG. 4. Spectral densities of estimated AR approximating $\operatorname{GARMA}(0, \delta, 0)$ models with different lag orders. 
as the Wolfer's sunspots numbers. The meta-analytical comparison was performed with established model results available in the literature; the optimal $m$ value was established by performing a rolling pseudo-real time forecasting experiment and by choosing the value that minimised the one-step-ahead mean square forecast error (see Dissanayake, Peiris and Proietti, 2016, for further details). These developments motivated the authors to apply the methodology to seasonal processes, which is the topic of Section 4.3.

\subsection{Seasonal Fractional Gegenbauer Processes}

The presence of long range dependence in the seasonal behaviour is a well-established phenomenon for environmental and economic time series. It has been observed in riverflows (Montanari, Rosso and Taqqu, 2000), monetary aggregates (see Porter-Hudak, 1990), time series of revenues (Ray, 1993), inflation rates (Hassler and Wolters, 1995), quarterly gross national product and shipping data (Ooms, 1995).

Several statistical modelling methodologies have been developed, among which we mention the fractional Gaussian noise process of Abrahams and Dempster (1979), the seasonal fractionally integrated autoregressive moving average (SARFIMA) model of Porter-Hudak (1990), the flexible seasonal fractionally integrated process (flexible ARFISMA) of Hassler (1994), the $k$-GARMA process of Woodward, Cheng and Gray (1998), the seasonal long range dependent process of Palma and Chan (2005), the seasonal fractionally integrated process of Reisen, Rodrigues and Palma (2006) and the seasonal ARFIMA model of Bisognin and Lopes (2009). Statistical inference for seasonal long memory processes has been dealt with by Giraitis and Leipus (1995), Chung (1996), Arteche and Robinson (2000), Velasco and Robinson (2000), Giraitis, Hidalgo and Robinson (2001), Palma (2007), Arteche (2007), Koopman, Ooms and Carnero (2007), Bisognin and Lopes (2009), Hsu and Tsai (2009) and Arteche (2012).

Let $s$ denote the seasonal period (e.g., $s=7$ for a weekly seasonal component in daily data). The seasonal changes are defined as $X_{t}-X_{t-s}=\left(1-B^{s}\right) X_{t}$. The seasonal lag polynomial $\left(1-B^{s}\right)$ has $s^{\prime}$ roots,

$$
\zeta_{k}=\cos \left(\omega_{k}\right)+\imath \sin \left(\omega_{k}\right)
$$

where $\omega_{k}=2 k \pi / s, k=0,1,2, \ldots, s^{\prime}-1$.
This implies the following factorization:

$$
\begin{aligned}
& \left(1-B^{s}\right) \\
& =(-1)^{s+1} \prod_{k=0}^{s-1}\left(\zeta_{k}-B\right) \\
& =\left\{\begin{array}{c}
(1-B) \prod_{k=1}^{(s-1) / 2}\left(1-2 \cos \left(\omega_{k}\right) B+B^{2}\right) \\
s \text { is odd, } \\
(1-B)(1+B) \prod_{k=1}^{(s / 2)-1}\left(1-2 \cos \left(\omega_{k}\right) B+B^{2}\right) \\
s \text { is even. }
\end{array}\right.
\end{aligned}
$$

A seasonal fractional Gegenbauer process of order $\delta_{s}$ is defined as

$$
\left(1-B^{s}\right)^{\delta_{s}} X_{t}=\epsilon_{t}, \quad \epsilon_{t} \sim \mathrm{WN}\left(0, \sigma^{2}\right) .
$$

The seasonal behaviour is due to the presence of $\lfloor s / 2\rfloor$ factors representing Gegenbauer polynomials defined at the frequencies $\omega_{k}=2 \pi k / s, k=1, \ldots,\lfloor s / 2\rfloor$, characterised by the same fractional integration order. A possible generalization allows for a Gegenbauer factor with angular frequency $\omega_{0}$ :

$$
\left(1-B^{s}\right)^{\delta_{s}}\left(1-2 u B+B^{2}\right)^{\delta} X_{t}=\epsilon_{t} .
$$

The extension of the state space modelling approach to seasonal GARMA processes can be performed by considering the $\mathrm{AR}(m)$ or $\mathrm{MA}(m)$ approximations to (4.3), obtained by truncating at $m$ th lag the infinite polynomials

$$
\left(1-B^{s}\right)^{\delta_{s}}\left(1-2 u B+B^{2}\right)^{\delta}=\sum_{j=0}^{\infty} \pi_{j} B^{j},
$$

and

$$
\left(1-B^{s}\right)^{-\delta_{s}}\left(1-2 u B+B^{2}\right)^{-\delta}=\sum_{j=0}^{\infty} \psi_{j} B^{j} .
$$

\section{MODELLING THE PERSISTENCE IN THE CONDITIONAL VARIANCE}

An important generalization of GARMA processes aims at modelling the conditional heteroscedasticity of the error process. This is relevant for the analysis and interval and density forecasting of electricity prices.

Most econometric models dealing with the persistence in the conditional variance are based on the 
class of generalized autoregressive conditional heteroscedasticity (GARCH) models. When the conditional mean is modelled via a $\operatorname{GARMA}(p, \delta, q)$ process, as in Dissanayake and Peiris (2011), the following $\operatorname{GARMA}(p, \delta, q)-\operatorname{GARCH}(r, s)$ representation arises:

$$
\begin{aligned}
& \phi(B)\left(1-2 u B+B^{2}\right)^{\delta} X_{t}=\theta(B) \epsilon_{t}, \\
& \epsilon_{t}=\sigma_{t} \varepsilon_{t}, \quad \varepsilon_{t} \sim \text { i.i.d. } \mathrm{N}(0,1), \\
& \sigma_{t}^{2}=\alpha_{0}+\sum_{j=1}^{r} \alpha_{j} \epsilon_{t-j}^{2}+\sum_{j=1}^{s} \beta_{j} \sigma_{t-j}^{2},
\end{aligned}
$$

with $\alpha_{0}>0, \alpha_{j} \geq 0, j=1, \ldots, r, \beta_{j} \geq 0, j=$ $1, \ldots, s$.

A unique member of this class, named as the ARFIMA $(0,2 \delta, 0)-\operatorname{GARCH}(r, s)$ model, was studied in detail by Ling and $\mathrm{Li}$ (1997), who have shown that there exists a unique $\mathcal{F}_{t}$-measurable second-order stationary solution $\left\{\varepsilon_{t}, X_{t}\right\}$, where $\mathcal{F}_{t}$ is the filtration generated by the history of the process.

Dissanayake, Peiris and Proietti (2014) extended the theory to the more general $\operatorname{GARMA}(0, \delta, 0)-$ $\operatorname{GARCH}(r, s)$ case, showing that, under the assumptions $|u|<1,0<\delta<\frac{1}{2}$ and $\sum_{i=1}^{r} \alpha_{i}+\sum_{j=1}^{s} \beta_{j}<1$, there exists an $\mathcal{F}_{t}$-measurable second-order stationary solution $\left\{\epsilon_{t}, X_{t}\right\}$ with causal representations

$$
\epsilon_{t}=\varepsilon_{t}\left\{\alpha_{0}+\sum_{j=1}^{\infty} D^{T}\left(\prod_{i=1}^{j} A_{t-i}\right) \mathcal{E}_{t-j}\right\}^{1 / 2} \quad \text { a.s. }
$$

and

$$
\begin{aligned}
X_{t}= & \sum_{k=0}^{[M / 2]}(-1)^{k} \\
& \cdot \frac{(2 u)^{M-2 k}(\delta+M-k) !}{k !(\delta-1) !(M-2 k) !} \epsilon_{t-k} \quad \text { a.s., }
\end{aligned}
$$

where $\mathcal{E}_{t}=\left(\alpha_{0} \varepsilon_{t}^{2}, 0, \ldots 0, \alpha_{0}, 0, \ldots, 0\right)^{\prime}$, an $(r+s) \times 1$ vector with nonzero first and $(r+1)$ thcomponents, $M$ is a fixed integer based on the required number of binomial coefficients, $D=\left(\alpha_{1}, \ldots, \alpha_{r}, \beta_{1}, \ldots, \beta_{s}\right)^{\prime}$, and

$$
A_{t}=\left[\begin{array}{ccc|ccc}
\alpha_{1} \varepsilon_{t}^{2} & \ldots & \alpha_{r} \varepsilon_{t}^{2} & \beta_{1} \varepsilon_{t}^{2} & \ldots & \beta_{s} \varepsilon_{t}^{2} \\
& I_{(r-1) \times(r-1)} & O_{(r-1) \times 1} & & O_{(r-1) \times s} & \\
\hline \alpha_{1} & \ldots & \alpha_{r} & \beta_{1} & \ldots & \beta_{s} \\
& O_{(s-1) \times r} & & I_{(s-1) \times(s-1)} & O_{(s-1) \times 1} &
\end{array}\right],
$$

and $I_{r \times r}$ is the $r \times r$ identity matrix.

Additionally, letting $\left\{X_{t, m}\right\}$ denote the $\operatorname{MA}(m)$ approximating process, $X_{t, m}=\sum_{k=0}^{m} C_{k} \epsilon_{t-k}$. Under the above assumptions and suitable regularity conditions,
Dissanayake, Peiris and Proietti (2014) show the convergence of $X_{t, m}$ to $X_{t}$. This opens the way to inference via the state space approach discussed in Section 4, suitably extended to handle conditional heteroscedasticity. Table 2 reports the results of a Monte Carlo experiment aiming at assessing the accuracy of the quasi maximum likelihood estimates based on the state space approach. The results are based on 1000 replications from a $\operatorname{GARMA}(0, \delta, 0)-\operatorname{GARCH}(1,1)$ process with $\delta=0.3, u=0.8, \alpha_{0}=0.4, \alpha_{1}=0.3$, $\beta_{1}=0.3$. The order of the MA and AR approximation have been chosen each time according to a rolling forecasting experiment as the value that is optimal for out of sample predictability.

An interesting fact that was observed is that the performance of the estimator of the memory parameter and the selection of the optimal $m$ th order approximation is unaffected by the volatility clustering in $\varepsilon_{t}$.

Another use of Gegenbauer processes is in modelling the long range dependence of the volatility of financial time series. Several studies report evidence of long memory in empirical volatility returns, as illustrated by Robinson (1991), Shephard (1996), Lobato and Savin (1998) and Baillie (1996). A new class of GIGARCH was introduced by Guegan (2000) to model volatility with generalized fractional integration. McAleer and Medeiros (2008) proposed a flexible model to describe nonlinearities and long memory in time series dynamics for the purpose of forecasting volatility. Lieberman and Phillips (2008) have provided some analytical explanations for long memory behaviour that has been observed in realized volatility. An elegant and parsimonious way of approximating the long memory behaviour of time series of realized volatility measures through a restricted autoregressive model was proposed by Corsi (2009).

\section{TESTING GARMA $(0, \delta, 0)$ PROCESSES}

A stochastic process $X_{t}$ is said to be integrated of order $\delta$, denoted $X_{t} \sim I(\delta)$, if $(1-B)^{\delta} X_{t}$ is a stationary process with absolutely summable autocorrelation function. This definition encompasses that of an $I(1)$ process, which is such that $(1-B) X_{t}$ is stationary and short memory. Also, a stationary short memory process will be said to be integrated of order zero, denoted $X_{t} \sim I(0)$.

The problem of testing whether $X_{t} \sim I(1)$ versus the alternative $X_{t} \sim I(0)$ is illustrated by Phillips and Xiao (1998). It is often found that both the null and the alternative hypotheses are rejected, suggesting that many 
TABLE 2

Monte Carlo simulation of a $\operatorname{GARMA}(0, \delta, 0)-\operatorname{GARCH}(1,1)$ process with $\delta=0.3, u=0.8, \alpha_{0}=0.4, \alpha_{1}=0.3, \beta=0.3$. Accuracy of the QMLE parameter estimates, based on 1000 replications (SE $=$ Standard Error, MSE $=$ Mean Square Error)

\begin{tabular}{|c|c|c|c|c|c|c|c|c|c|c|}
\hline & \multicolumn{10}{|c|}{$T=1000$} \\
\hline & \multicolumn{5}{|c|}{ MA approximation } & \multicolumn{5}{|c|}{ AR approximation } \\
\hline & $\hat{\delta}$ & $\hat{u}$ & $\hat{\alpha_{0}}$ & $\hat{\alpha_{1}}$ & $\hat{\beta}_{1}$ & $\hat{\delta}$ & $\hat{u}$ & $\hat{\alpha_{0}}$ & $\hat{\alpha_{1}}$ & $\hat{\beta}_{1}$ \\
\hline Mean & 0.306 & 0.788 & 0.430 & 0.264 & 0.296 & 0.305 & 0.792 & 0.424 & 0.273 & 0.293 \\
\hline SE & 0.082 & 0.130 & 0.149 & 0.180 & 0.098 & 0.081 & 0.126 & 0.148 & 0.184 & 0.096 \\
\hline \multirow[t]{2}{*}{ MSE } & 0.006 & 0.017 & 0.023 & 0.033 & 0.009 & 0.006 & 0.016 & 0.022 & 0.034 & 0.009 \\
\hline & \multicolumn{10}{|c|}{$T=2000$} \\
\hline Mean & 0.299 & 0.804 & 0.403 & 0.296 & 0.300 & 0.299 & 0.804 & 0.403 & 0.296 & 0.299 \\
\hline SE & 0.018 & 0.022 & 0.050 & 0.063 & 0.034 & 0.020 & 0.028 & 0.052 & 0.065 & 0.034 \\
\hline MSE & 0.0004 & 0.0008 & 0.002 & 0.004 & 0.001 & 0.0003 & 0.0005 & 0.002 & 0.004 & 0.001 \\
\hline
\end{tabular}

series are not well represented by both the $I(0)$ and I (1) hypotheses.

The definition can be extended to allow for integration at any frequency in the range $(0, \pi)$. In particular, we will say that $X_{t}$ is integrated of order $\delta$ at the frequency $\omega_{0}$, denoted $I\left(\delta, \omega_{0}\right)$ if $\left(1-2 u B+B^{2}\right)^{\delta} X_{t}$ admits a stationary short memory representation, where $u=\cos \left(\omega_{0}\right)$.

We will focus on the following testing problems:

1. Test $H_{0}: X_{t} \sim I(2 \delta, 0)$, versus $H_{1}: X_{t} \sim I(\delta$, $\left.\omega_{0}\right), \omega_{0} \in(0, \pi)$.

2. Test $H_{0}: X_{t} \sim I\left(0.5, \omega_{0}\right)$ versus $H_{1}: X_{t} \sim I(\delta$, $\left.\omega_{0}\right), \delta<0.5$.

We can equivalently reparameterize the first case in terms of $u$, with $H_{0}: u=1$ and $H_{1}:|u|<1$. Under the null, the process is a standard long memory process with fractional integration order $2 \delta$, whereas under the alternative it is a Gegenbauer process integrated at an angular frequency different from zero. Hence, the first case is a test for the presence of a stochastic persistent cycle. In the second case, we test whether the Gegenbauer process nonstationary at the frequency $\omega_{0}$, versus the alternative that it is stationary, with fractional order $\delta<0.5$.

Chung (1996) performed these testing problems, basing the inference on a conditional sum of squares estimation method. The approach that is taken by Dissanayake, Peiris and Proietti (2015) and Dissanayake et al. (2015) is based on the quasilikelihood ratio (QLR) test arising from the maximized likelihood of the MA or AR approximations both under the null and the alternative. The QLR approach can be justified by the theoretical constructs of equations (13), (14) and the description of Section 4.1.

Consider the $\operatorname{GARMA}(0, \delta, 0)$ process model defined in (1), with $\epsilon_{t} \sim$ i.i.d. $\mathrm{N}\left(0, \sigma^{2}\right)$. The Gegenbauer polynomial could be written as

$$
1-2 u B+B^{2}=\left[2(u-1) \varphi(\Delta-1)+\Delta^{2}\right],
$$

where $\Delta=(1-B)$, so that we can reparameterize the process as follows:

$$
\left[2(u-1)(\Delta-1)+\Delta^{2}\right]^{\delta} X_{t}=\epsilon_{t} .
$$

The test of $H_{0}: u=1$ (under which the true model is $\Delta^{2 \delta} X_{t}=\epsilon_{t}$ ), versus $H_{1}: u<1$ [under which the process is integrated of order $\delta$ at the frequency $\omega_{0}=$ $\arccos (u)]$, is

$$
L R_{T}^{u}=\max \left[\ell_{\sigma_{m}^{2}}(\delta, u)\right]-\max \left[\ell_{\sigma_{m}^{2}}(\delta, 1)\right],
$$

where the profile likelihood is given in (17). The distribution of the test statistic is nonstandard, as $u$ lies on the boundary of the parameter space; see Dissanayake, Peiris and Proietti (2015) for more details.

As far as the test of nonstationarity is concerned, $H_{0}: \delta=0.5$, the quasi-likelihood ratio test statistic is

$$
L R_{T}^{\delta}=\max \left[\ell_{\sigma_{m}^{2}}(\delta, u)\right]-\max \left[\ell_{\sigma_{m}^{2}}(1 / 2, u)\right],
$$

Again, the distribution of the test statistic is nonstandard and we refer to Dissanayake et al. (2015) for further details.

Following Jansson and Nielsen (2012), Dissanayake, Peiris and Proietti (2015) and Dissanayake et al. (2015) explore the power properties of their quasi-likelihood ratio test. Their evidence is that the power of the tests are reasonable and comparable with those presented in 
Beaumont and Ramachandran (2001). For testing the fractional order of seasonal and nonseasonal unit roots of long memory processes, see Ferrara, Guegan and Lu (2010).

The QMLE rests on the Gaussian assumption and the ability to select the optimal order of the approximation. In the case of GARMA processes with GARCH disturbances, an important issue would be assessing the robustness of the estimation and the testing of deviation procedures from Gaussianity. It does not come under the purview of this paper and would be a worthwhile exercise for a proactive researcher in statistics or econometrics.

\section{CONCLUDING REMARKS}

The facts provided in this review highlight certain gaps in the literature linked with fractionally differenced long memory Gegenbauer processes. Relevant model formulation, state space based estimation and a related nearly efficient unit root testing procedure have been investigated. New results have been presented in the discussion of this paper to encourage further research within the statistical and econometric communities.

\section{ACKNOWLEDGEMENTS}

Authors thank the Editor, Associate Editor and two anonymous reviewers for their excellent comments and fruitful suggestions. They would also like to thank Jackie Nicholas of the Mathematics Learning Centre, University of Sydney, for her meticulous proof reading that enabled them to improve and enhance the readability of this manuscript. The second author acknowledges the support received from the Department of Statistics, University of Colombo and the School of Business, NSBM Green University, Sri Lanka, in preparing the final version of the manuscript.

\section{REFERENCES}

Abrahams, M. and Dempster, A. (1979). Research on seasonal analysis. Progress report on the asa/census project on seasonal adjustment. Technical report. Dept. Statistics, Harvard Univ., Boston, MA.

ANDĚL, J. (1986). Long memory time series models. Kybernetika (Prague) 22 105-123. MR0849684

Anderson, B. D. O. and Moore, J. B. (1979). Optimal Filtering. Prentice-Hall, New York.

Anh, V. V., Angulo, J. M. and Ruiz-MedinA, M. D. (1999). Possible long-range dependence in fractional random fields. $J$. Statist. Plann. Inference 80 95-110. MR1713795
ANH, V. V., Lunney, K. and PEIRIS, S. (1997). Stochastic models for characterisation and prediction of time series with longrange dependence and fractality. Environ. Model. Softw. 12 6773.

AoKI, M. (1990). State Space Modeling of Time Series, 2nd ed. Springer, Berlin. MR1073171

ARTECHE, J. (2007). The analysis of seasonal long memory: The case of Spanish inflation. Oxf. Bull. Econ. Stat. 69 749-772.

ARTECHE, J. (2012). Standard and seasonal long memory in volatility: An application to Spanish inflation. Empir. Econ. 42 693-712.

ARTEChe, J. and Robinson, P. M. (2000). Semiparametric inference in seasonal and cyclical long memory processes. J. Time Series Anal. 21 1-25. MR1766171

BAILliE, R. T. (1996). Long memory processes and fractional integration in econometrics. J. Econometrics 73 5-59. MR1410000

Baillie, R. T., Bollerslev, T. and Mikkelsen, H. O. (1996). Fractionally integrated generalized autoregressive conditional heteroskedasticity. J. Econometrics 74 3-30. MR1409033

BEAUMONT, P. and RAMACHANDRAN, R. (2001). Robust estimation of GARMA model parameters with an application to cointegration among interest rates of industrialized countries. Comput. Econ. 17 179-201.

BERAN, J. (1992). Statistical methods for data with long-range dependence. Statist. Sci. 7 404-416.

BERAN, J. (1993). Fitting long-memory models by generalized linear regression. Biometrika $\mathbf{8 0} 817-822$. MR1282790

BERAN, J. (1994). Statistics for Long-Memory Processes. Monographs on Statistics and Applied Probability 61. Chapman \& Hall, New York. MR1304490

Beran, J., Feng, Y., Ghosh, S. and KuliK, R. (2013). LongMemory Processes: Probabilistic Properties and Statistical Methods. Springer, Heidelberg. MR3075595

Bisognin, C. and Lopes, S. R. C. (2009). Properties of seasonal long memory processes. Math. Comput. Modelling $491837-$ 1851. MR2532092

BollersLEV, T. (1986). Generalized autoregressive conditional heteroskedasticity. J. Econometrics 31 307-327. MR0853051

Box, G. E. P. and Jenkins, G. M. (1970). Times Series Analysis. Forecasting and Control. Holden-Day, San Francisco, CA. MR0272138

Brockwell, P. J. and DAVIS, R. A. (1991). Time Series: Theory and Methods, 2nd ed. Springer, New York. MR1093459

BRoCKWELL, P. J. and DAVIS, R. A. (1996). Introduction to Time Series and Forecasting. Springer, New York. MR1416563

Chan, N. H. and PALMA, W. (1998). State space modeling of long-memory processes. Ann. Statist. 26 719-740. MR1626083

Chan, N. H. and PALMA, W. (2006). Estimation of long-memory time series models: A survey of different likelihood-based methods. Adv. Econom. 20 89-121. MR2498114

Chung, C.-F. (1996). A generalized fractionally integrated autoregressive moving-average process. J. Time Series Anal. 17 111-140. MR1381168

Corsi, F. (2009). A simple approximate long-memory model of realized volatility. J. Financ. Econom. 7 174-196.

Dissanayake, G. S. and PEIRIS, M. S. (2011). Generalized fractional processes with conditional heteroskedasticity. Sri Lankan J. Appl. Stat. 12 1-12. 
Dissanayake, G. S., Peiris, M. S. and Proietti, T. (2014). Estimation of generalized fractionally differenced processes with conditionally heteroskedastic errors. In International Work Conference on Time Series, Proceedings ITISE 2014 (I. R. Ruiz and G. R. Garcia, eds.). Copicentro Granada S L 871-890.

Dissanayake, G. S., Peiris, M. S. and Proietti, T. (2015). State space modeling of seasonal Gegenbauer processes with long memory. Working Paper, School of Mathematics and Statistics, Univ. Sydney, Australia.

Dissanayake, G. S., Peiris, M. S. and Proietti, T. (2016). State space modeling of Gegenbauer processes with long memory. Comput. Statist. Data Anal. 100 115-130. MR3505794

Dissanayake, G. S., Peiris, M. S., Proietti, T. and WANG, Q. (2015). Nearly efficient testing and asymptotics of a long memory GARMA $(0, \delta, 0)$ process, Working Paper, School of Mathematics and Statistics, Univ. Sydney, Australia.

Dolado, J. J., Gonzalo, J. and MAYORAL, L. (2002). A fractional Dickey-Fuller test for unit roots. Econometrica 701963 2006. MR1925162

Durbin, J. and Koopman, S. J. (2001). Time Series Analysis by State Space Methods. Oxford Statistical Science Series 24. Oxford Univ. Press, Oxford. MR1856951

ENGLE, R. F. (1982). Autoregressive conditional heteroscedasticity with estimates of the variance of United Kingdom inflation. Econometrica 50 987-1007. MR0666121

Erdelyi, A., Magnus, W., Oberhettinger, F. and TriCOMI, F. G. (1953). Higher Transcendental Functions, Vol. II, Bateman Manuscript Project. McGraw-Hill. New York.

FERrarA, L. and GUEGAN, D. (2001). Forecasting with k-factor Gegenbauer processes: Theory and applications. J. Forecast. 20 581-601.

Ferrara, L., Guegan, D. and Lu, Z. (2010). Testing fractional order of long memory processes: A Monte Carlo study. Comm. Statist. Simulation Comput. 39 795-806. MR2785667

Giraitis, L., Hidalgo, J. and Robinson, P. M. (2001). Gaussian estimation of parametric spectral density with unknown pole. Ann. Statist. 29 987-1023. MR1869236

Giraitis, L., Koul, H. L. and Surgailis, D. (2012). Large Sample Inference for Long Memory Processes. Imperial College Press, London. MR2977317

GiRAITIS, L. and LEIPUS, R. (1995). A generalized fractionally differencing approach in long-memory modeling. Liet. Mat. Rink. 35 65-81. MR1357812

GonÇALVES, E. (1987). Une généralisation des processus ARMA. Ann. Écon. Stat. 5 109-145. MR0907560

Gould, H. W. (1974). Coefficient identities for powers of Taylor and Dirichlet series. Amer. Math. Monthly 81 3-14. MR0340038

Gradshteyn, I. S. and RYZHiK, I. M. (1980). Tables of Integrals Series and Products. Academic Press, New York.

Granger, C. W. J. and JoyeuX, R. (1980). An introduction to long-memory time series models and fractional differencing. $J$. Time Series Anal. 1 15-29. MR0605572

Grassi, S. and SAntucci de Magistris, P. (2014). When long memory meets the Kalman filter: A comparative study. Comput. Statist. Data Anal. 76 301-319. MR3209443

Gray, H. L., Zhang, N.-F. and Woodward, W. A. (1989). On generalized fractional processes. J. Time Series Anal. 10233 257. MR1028940
Gray, H. L., Zhang, N.-F. and WoOdWARd, W. A. (1994). A correction: "On generalized fractional processes" [J. Time Ser. Anal. 10 (1989), no. 3, 233-257; MR1028940 (90m:62208)]. J. Time Series Anal. 15 561-562. MR1292167

GUEGAN, D. (2000). A new model: The k-factor GIGARCH process. J. Signal Process. 4 265-271.

GuÉGAN, D. (2005). How can we define the concept of long memory? An econometric survey. Econometric Rev. 24 113-149. MR2190313

HARVEY, A. C. (1989). Forecasting, Structural Time Series Models and the Kalman Filter. Cambridge Univ. Press, Cambridge.

Harvey, A. C. and Proietti, T., eds. (2005). Readings in Unobserved Components Models. Oxford Univ. Press, Oxford. MR2381922

HASSLER, U. (1994). (Mis)specification of long memory in seasonal time series. J. Time Series Anal. 15 19-30. MR1256854

HASSLER, U. and Wolters, J. (1994). On the power of unit root tests against fractional alternatives. Econom. Lett. 45 1-5.

HASSLER, U. and WOLTERS, J. (1995). Long memory in inflation rates: International evidence. J. Bus. Econom. Statist. 13 37-45.

Hosking, J. R. M. (1981). Fractional differencing. Biometrika 68 165-176. MR0614953

HSU, N.-J. and TSAI, H. (2009). Semiparametric estimation for seasonal long-memory time series using generalized exponential models. J. Statist. Plann. Inference 139 1992-2009. MR2497555

JAnsson, M. and Nielsen, M. Ø. (2012). Nearly efficient likelihood ratio tests of the unit root hypothesis. Econometrica $\mathbf{8 0}$ 2321-2332. MR3013726

KALMAN, R. E. (1961). A new approach to linear filtering and prediction problems. Trans. Am. Soc. Mech. Eng. 83D 35-45.

KALMAN, R. E. and BUCY, R. S. (1961). New results in linear filtering and prediction theory. Trans. Am. Soc. Mech. Eng. 83 95-108. MR0234760

Koopman, S. J., Ooms, M. and CARnero, M. A. (2007). Periodic seasonal Reg-ARFIMA-GARCH models for daily electricity spot prices. J. Amer. Statist. Assoc. 102 16-27. MR2345529

Lieberman, O. and Phillips, P. C. B. (2008). Refined inference on long memory in realized volatility. Econometric Rev. 27 254-267. MR2424814

LING, S. and LI, W. K. (1997). On fractionally integrated autoregressive moving-average time series models with conditional heteroscedasticity. J. Amer. Statist. Assoc. 92 1184-1194. MR1482150

Lobato, I. N. and SAVIN, N. E. (1998). Real and spurious long-memory properties of stock-market data. J. Bus. Econom. Statist. 16 261-283. MR1648533

LU, Z. and GUEGAN, D. (2011). Estimation of time-varying long memory parameter using wavelet method. Comm. Statist. Simulation Comput. 40 596-613. MR2783896

McAleer, M. and Medeiros, M. C. (2008). A multiple regime smooth transition heterogeneous autoregressive model for long memory and asymmetries. J. Econometrics 147104 119. MR2472985

Montanari, A., Rosso, R. and Taqqu, M. S. (2000). A seasonal fractional ARIMA modelapplied to Nile river monthly flows at Aswan. Water Resour. Res. 36 1249-1259.

Ohanissian, A., Russell, J. R. and Tsay, R. S. (2008). True or spurious long memory? A new test. J. Bus. Econom. Statist. 26 161-175. MR2420145 
Ooms, M. (1995). Flexible seasonal long memory and economic time series. Technical Report EI-9515/A. Econometric Institute, Erasmus Univ., Rotterdam.

Oppenheim, G. and Viano, M.-C. (2004). Aggregation of random parameters Ornstein-Uhlenbeck or AR processes: Some convergence results. J. Time Series Anal. 25 335-350. MR2062677

PALMA, W. (2007). Long-Memory Time Series: Theory and Methods. Wiley-Interscience, Hoboken, NJ. MR2297359

Palma, W. and Chan, N. H. (2005). Efficient estimation of seasonal long-range-dependent processes. J. Time Series Anal. 26 863-892. MR2203515

Pearlman, J. G. (1980). An algorithm for the exact likelihood of a high-order autoregressive-moving average process. Biometrika 67 232-233. MR0570525

PEIRIS, M. S. (2003). Improving the quality of forecasting using generalized AR models: An application to statistical quality control. Stat. Methods 5 156-171. MR2198741

PeIRIS, S., Allen, D. and PEIRIS, U. (2005). Generalized autoregressive models with conditional heteroscedasticity: An application to financial time series modeling. In Proceedings of the Workshop on Research Methods: Statistics and Finance 75-83.

PEIRIS, S. and ASAI, M. (2016). Generalized fractional processes with long memory and time dependent volatility revisited. Econometrics 437.

Peiris, S. and ThaVANESWARAN, A. (2007). An introduction to volatility models with indices. Appl. Math. Lett. 20 177-182. MR2283907

Phillips, P. C. B. and XIAO, Z. (1998). A primer on unit root testing. J. Econ. Surv. 12 423-470.

PorTER-HudAK, S. (1990). An application of the seasonal fractionally differenced model to the monetary aggregates. J. Amer. Statist. Assoc. 85 338-344.

Rainville, E. D. (1960). Special Functions. The Macmillan Co., New York. MR0107725

RAY, B. K. (1993). Modeling long-memory processes for optimal long-range prediction. J. Time Series Anal. 14 511-525. MR1243579

Reisen, V. A., Rodrigues, A. L. and Palma, W. (2006). Estimation of seasonal fractionally integrated processes. Comput. Statist. Data Anal. 50 568-582. MR2201879
RoBinson, P. M. (1991). Testing for strong serial correlation and dynamic conditional heteroskedasticity in multiple regression. J. Econometrics 47 67-84. MR1087207

SHEPHARD, N. (1996). Statistical aspects of ARCH and stochastic volatility. In Time Series Models: In Econometrics, Finance and Other Fields (D. R. Cox, D. B. Hinkley, and O. E. BarndorffNielsen, eds.), Chapman \& Hall, London.

ShITAN, M. and PEIRIS, S. (2008). Generalized autoregressive (GAR) model: A comparison of maximum likelihood and Whittle estimation procedures using a simulation study. Comm. Statist. Simulation Comput. 37 560-570. MR2749969

ShItAN, M. and PEIRIS, S. (2009). On properties of the second order generalized autoregressive GAR(2) model with index. Math. Comput. Simulation 80 367-377. MR2582119

ShITAN, M. and PEIRIS, S. (2013). Approximate asymptotic variance-covariance matrix for the Whittle estimators of GAR(1) parameters. Comm. Statist. Theory Methods 42 756770. MR3028974

SLUTSKY, E. (1927). The summation of random causes as the source of cyclic processes. Econometrica 5 105-146.

Spolia, S. K., Chandler, S. and O'Connor, K. M. (1980). An autocorrelation approach for parameter estimation of fractional order equal-root autoregressive models using hypergeometric functions. J. Hydrol. 47 1-18.

TAYLOR, A. M. R. (2005). Fluctuation tests for a change in persistence. Oxf. Bull. Econ. Stat. 67 207-230.

Velasco, C. and Robinson, P. M. (2000). Whittle pseudomaximum likelihood estimation for nonstationary time series. J. Amer. Statist. Assoc. 95 1229-1243. MR1804246

WANG, Q., Lin, Y.-X. and Gulati, C. M. (2003). Asymptotics for general fractionally integrated processes with applications to unit root tests. Econometric Theory 19 143-164. MR1965845

Woodward, W. A., Cheng, Q. C. and Gray, H. L. (1998). A $k$-factor GARMA long-memory model. J. Time Series Anal. 19 485-504. MR1650550

Yule, G. U. (1926). Why do we sometimes get nonsensecorrelations between time-series? - A study in sampling and the nature of time-series. J. Roy. Statist. Soc. 89 1-63. 